\title{
MicroRNA-based Targeted Therapeutics in Pancreatic Cancer
}

\author{
NILGUN GURBUZ ${ }^{1}$ and BULENT OZPOLAT ${ }^{2}$ \\ ${ }^{1}$ Department of Medical Biology, School of Medicine, Suleyman Demirel University, Isparta, Turkey; \\ ${ }^{2}$ Department of Experimental Therapeutics, MD Anderson Cancer Center, \\ The University of Texas, Houston, TX, U.S.A.
}

\begin{abstract}
The discovery during the last decade of microRNAs (miRs, miRNA) and their role in regulating normal physiological processes as well as in the pathogenesis of human tumors has been a revolutionary development in molecular oncology. miRNAs activating or inhibiting oncogenic molecular pathways that are involved in tumorigenesis, cell progression, invasion, angiogenesis and metastasis are now considered of major impact in many cancer types. miRNA-based therapeutics that inhibit the levels of oncogenic miRNAs (oncomiRs) or elevate tumor suppressor miRs have enormous potential as molecular therapeutic targets. Thus, the development of new targeted cancer therapies based on miRNAs promise to revolutionize cancer treatment due to their increased efficacy compared to conventional chemoradiation-based therapies and hopefully to lower levels of adverse effects.
\end{abstract}

A revolutionary development in molecular oncology during the last decade has been the discovery of the role of micro RNAs (miRNA) in the pathogenesis of tumors (1). miRNAs are small (18-25 nucleotides in length) non-coding RNA molecules that act as post-transcriptional regulators of gene expression by binding to the 3'-UTR of target genes (2). miRNAs are produced in the nucleus by the cleavage of 85 nucleotide-long hair-pin shaped pre-miRNAs by RNase III Dicer followed by their transportation to the cytoplasm by

This article is freely accessible online.

Correspondence to: Bulent Ozpolat, MD, Ph.D., Department of Experimental Therapeutics, Unit 422, The University of Texas MD Anderson Cancer Center, 1515 Holcombe Boulevard, Houston, TX 77030, U.S.A. Tel: +1 7135630166, Fax: +1 7137920362. E-mail: bozpolat@mdanderson.org

Key Words: miRNA, pancreatic cancer, targeted therapy, clinical trial, nanoparticle, review.
Exportin-5, pre-miRNAs are also cleaved from a larger precursor called pri-miRNA by the enzyme RNase III in the nucleus (2). miRNAs, together with the Argonaute family proteins, form the RNA-induced silencing complex (RISC), which suppresses the translation of mRNAs in the cytoplasm. The miRNAs are not specific to a single mRNA sequence and can regulate a set of different mRNAs, thus each mRNA can be regulated by a set of different miRNAs (3). miRNAs are secreted by cancer cells into the extracellular fluids and systemic circulation through exosomes, small 40-100 nm nano-sized cell-derived vesicles that are released from most cells can modulate the function of neighboring and distant cells and play an important role in intercellular communication process in tumor microenvironment (4).

Recent studies show that miRNAs regulate the expression of genes involved in many cellular processes including cell proliferation, differentiation, invasion, angiogenesis, drug resistance and apoptosis, all of which contribute to tumor growth and progression (2-4). miRNAs may function as oncogenes (oncomiRs) or tumor suppressors and their expression is often dysregulated in tumors. OncomiRs promote cancer development by negatively regulating tumor suppressor genes that control cell proliferation, migration/invasion or key mediators related to molecular mechanisms in cancer promotion $(2,3)$. Tumor suppressor miRNAs often inhibit the expression of oncogenes and genes that promote cell proliferation, invasion and tumorigenesis. Studies show that the expression of tumor suppressors often reduces, ceases/or is lost, while oncomiRs expression increases in cancer cell lines as well as within patients' tumors (3). Taken together, these data suggest that the expression profiles of certain miRNA molecules may become useful biomarkers in cancer diagnostics (4). In addition, miRNA-based therapies inhibiting oncomiRs or activating of tumor suppressor miRs, could be a powerful tool for cancer prevention and therapy (5-8).

Pancreatic cancer is one of the deadliest types of cancer, with approximately $2 \%$ of patient survivors within 5-years following their diagnosis (9). Because of that late-stage 
Table I. miRNAs differentially expressed in PDAC samples with a potential to be used as biomarkers for PDAC.

Pancreatic cancer

\begin{tabular}{ll}
\hline Up-regulated miRs & Down-regulated miRs \\
\hline miR10b,miR-21, miR23a, miR-31, miR-143, miR-145, miR-146a, & miR-29c, miR -30a, miR-34a, miR -96, -130b, miR -141, \\
miR-150, miR-155, miR-181a/b/c, miR-196a/b, miR-21, miR-210, & miR -148a, miR-148b, miR-216, miR-217, miR-375 and miR -494 \\
miR-221, miR-222, miR-223, miR-376a and miR-301 &
\end{tabular}

diagnosis and the limited chemotherapeutic opportunities, the prognosis of pancreatic cancer is extremely poor and median survival in the US and EU is about 6 months (9). Pancreatic ductal adenocarcinomas (PDAC) derived from the exocrine pancreas account for $95 \%$ of pancreatic cancers. Current chemotherapy of PDAC is frequently insufficient, leading to 6 months of patient survival on average (10). Therefore, there is an urgent need for a better understanding of the molecular mechanisms behind the role of miRNAs in the behavior of pancreatic cancer that can lead to the identification of novel molecular targets and development of novel targeted therapies to improve patient survival.

A growing body of evidence based on miRNA profiling from in vitro models as well as from PDAC patient samples, indicate that a large number of miRNAs is deregulated in pancreatic cancer (11-13). About 439 miRNAs have been found to be differentially expressed in PDAC samples (based on a comparison between 538 PDAC and 206 control samples), indicating some of them as useful biomarkers for PDAC (11) (Table I). The most significant overexpressed miRNAs in pancreatic cancer include miR-10b, miR-21, miR-23a, miR-31, miR-100miR-143, miR-145, miR-146a, miR-150, miR-155, miR-181a/b/c, miR-196a/b, miR-21, miR-210, miR-221, miR-222, miR-223, miR-376a and miR301. On the other hand, miR-148a, miR-217, miR-34a and miR-375 are frequently down-regulated in PDAC (11-14).

Currently, miR-based targeted therapeutic strategies in cancer involve the therapeutic administration of: i) tumor suppressor miRNA mimics and ii) inhibitors of miRNAs, known as anti-miRs/antagomiRs, that can block the effect of oncomiRs (5-7). Double-stranded miRNA mimics aim to restore the loss of miRNA expression, whereas anti-miRs, single stranded anti-sense oligonucleotides (complementary to target miRNA molecules), are designed to target mRNAs that bear oncogenic properties, affect drug resistance and cancer stem cell survival and maintenance (5-7). The therapeutic use of miR mimics and antimiRs/ antagomiRs in cancer clinical trials require an improvement of their binding affinity for the target mRNAs, their stability in the blood circulation, their pharmacokinetics as well as their delivery in the cancer cells/tissue. One of these modifications involves the addition of a methyl or a thiol group in the passenger strand of nucleotides or in the RNA backbone, to decrease their degradation rate in the circulation. Examples of chemical modifications present in anti-miRs include, the 2'-O-methyl-group-modification and the locked nucleic acid (LNA) modification, which can increase the serum stability to nucleases, improve pharmacological properties, such as increased serum stability and bioavailability as well as better pharmacokinetics profile with enhanced half-life and cellular uptake, leading to effective blocking onco-miRNAs both in vitro and in vivo tumor models (15-17). Importantly, the 2'$\mathrm{O}$-methoxyethyl modification also increases the specificity of the miRNA for its targets and it has been successfully used in human clinical trials with good safety records (18).

The specific delivery of RNA intervention therapeutics (siRNA, miR-mimics and anti-miRs/miR-inhibitors) into tumors still remains a major challenge. However, their administration within nano-vehicles/nanoparticles can improve some poor pharmacokinetic features (i.e. short halflife) and tumor-cell delivery (19-29).

miRNA-based therapeutics have been shown to be successful in preclinical animal studies (24-31) while some of them have entered human clinical trials for cancer and for other diseases $(32,33)$. The first ever miRNA-based clinical trial was Miravirsen, which has gone on to Phase II with the use of an LNA-based miRNA inhibitor, targeting miR-122 in liver cells infected with Hepatitis C virus (32). Another miRNA mimic drug, MRX34, has been used in a Phase I clinical trial as a lipid-based nanoparticle, to treat solid tumors, such as in the liver, but also for the treatment of hematologic malignancies (33). Unfortunately, this study was recently terminated due to the drug's side effects. Cobomarsen, an antimiR-155 inhibitor therapy, is ongoing for patients with adult T-cell leukemia/lymphoma and demonstrated greater than five months of clinical response with improvement in objective disease measurements (34). Despite all the successful preclinical studies with the pancreatic cancer models (27-31), miRNA-based gene therapy has not yet been developed to treat pancreatic cancer in humans. In an orthotopic model of pancreatic cancer using MiaPaca-2 cells, the systemic intravenous delivery of lipid nanoparticles carrying miR-34a mimics (a component of the p53 transcriptional network), or a miR-143/145 cluster (an inhibitor of KRAS and RREB1), 
has resulted in the decrease of tumor growth, CD44+ cell counts, and in the increase of tumor cell apoptosis (27). In addition to this report, the therapeutic delivery of miR-34awith nanocomplexes with a tumor-targeting and tumorpenetrating, bifunctional $\mathrm{CC} 9$ peptide, has been tested in a Panc-1 xenograft model and has revealed increased miR-34a levels and reduced mRNAs of Bcl-2, c-Myc, Cyclin D1 and E2F3 (28). The down-regulation of these major oncogenes has resulted in a significant inhibition of tumor growth, cycle progression, and migration while it has induced apoptosis of PDAC cells in this model (28). The nanoparticle-based delivery system for miR-150-NF, a tumor suppressive miRNA in PDAC, has led to increased intracellular delivery in vitro and has inhibited cell proliferation, clonogenicity and invasion of pancreatic cancer cells (29).

In conclusion, miRNA-based therapeutics are promising potential candidates as targeting agents against PDAC. Compared to siRNAs or small molecule inhibitors that target a single gene or protein, miRNAs are expected to be more successful than due to their potential to target multiple genes and several pathways at the same time. However, delivery of the miR-based therapeutics to target cancer cells and tumor tissues is a major challenge for successful, non-toxic and safe clinical applications in pancreatic cancer, as well as in other types cancer.

\section{Conflicts of Interest}

The Authors declare no conflict of interests.

\section{References}

1 Iorio MV and Croce CM: MicroRNAs in cancer: Small molecules with a huge impact. J Clin Oncol 27: 5848-5856, 2009.

$2 \mathrm{Ha} \mathrm{M}$ and Kim VN: Regulation of microRNA biogenesis. Nat Rev Mol Cell Biol 15: 509-524, 2014.

3 Croce CM: Causes and consequences of microRNA dysregulation in Cancer. Nat Rev Genet 10: 704-714, 2009.

4 Calin GA and Croce CM: MicroRNA signatures in human cancers. Nat Rev Cancer 6: 857-866, 2006.

5 Ling H, Fabbri $M$ and Calin GA: MicroRNAs and other noncoding RNAs as targets for anticancer drug development. Nat Rev Drug Discov 12: 847-865, 2013.

6 Cheng CJ, Bahal R, Babar IA, Pincus Z, Barrera F, Liu C, Svoronos A, Braddock DT, Glazer PM, Engelman DM, Saltzman WM and Slack FJ: MicroRNA silencing for cancer therapy targeted to the tumour microenvironment. Nature 518: 107-110, 2015.

7 Garzon R, Marcucci G and Croce CM: Targeting microRNAs in cancer: rationale, strategies and challenges. Nat Rev Drug Discov 9: 775-789, 2010.

8 Kong YW, Ferland-McCollough D, Jackson TJ and Bushell M: microRNAs in cancer management. Lancet Oncol 13: e249-58, 2012

9 Siegel R, Naishadham D and Jemal A: Cancer statistics, 2012. Cancer J Clin 62: 10-29, 2012.
10 Becker AE, Hernandez YG, Frucht $\mathrm{H}$ and Lucas AL: Pancreatic ductal adenocarcinoma: risk factors, screening, and early detection. World J Gastroenterol 20: 11182-11198, 2014.

11 Zhang Y, Li M, Wang H, Fisher WE, Lin PH, Yao Q and Chen C: Profiling of 95 microRNAs in pancreatic cancer cell lines and surgical specimens by real-time PCR analysis. World J Surg 33: 698-709, 2009.

12 Ma MZ, Kong X, Weng MZ, Cheng K, Gong W, Quan ZW and Peng CH: Candidate microRNA biomarkers of pancreatic ductal adenocarcinoma: Meta-analysis, experimental validation and clinical significance. J Exp Clin Cancer Res 32: 71, 2013.

13 Passadouro $M$ and Faneca $H$ : Managing Pancreatic Adenocarcinoma: A Special Focus in MicroRNA Gene Therapy. Int J Mol Sci 13: 17(5), 2016.

14 Jung KH, Zhang J, Zhou C1, Shen H, Gagea M, RodriguezAguayo C, Lopez-Berestein G, Sood AK and Beretta L: Differentiation therapy for hepatocellular carcinoma: Multifaceted effects of miR-148a on tumor growth and phenotype and liver fibrosis. Hepatology 63: 864-879, 2016.

15 Weiler J, Hunziker J and Hall J: Anti-miRNA oligonucleotides (AMOs): ammunition to target miRNAs implicated in human disease? Gene Ther 13: 496-502, 2006.

16 Krützfeldt J, Rajewsky N, Braich R, Rajeev KG, Tuschl T, Manoharan M, Stoffel M: Silencing of microRNAs in vivo with 'antagomirs'. Nature 438: 685-689, 2005.

17 Swayze EE, Siwkowski AM, Wancewicz EV, Migawa MT, Wyrzykiewicz TK, Hung G, Monia BP and Bennett CF: Antisense oligonucleotides containing locked nucleic acid improve potency but cause significant hepatotoxicity in animals. Nucleic Acids Res 35: 687-700, 2007.

18 Lennox KA and Behlke MA: Chemical modification and design of anti-miRNA oligonucleotides. Gene Ther 18: 1111-1120, 2011.

19 Pecot CV, Calin GA, Coleman RL, Lopez-Berestein G and Sood AK: RNA interference in the clinic: challenges and future directions. Nat Rev Cancer 11: 59-67, 2011.

20 Ozpolat B, Sood AK and Lopez-Berestein G: Nanomedicine based approaches for the delivery of siRNA in cancer. J Intern Med 267: 44-53, 2010.

21 Ozpolat B, Sood AK and Lopez-Berestein G: Liposomal siRNA nanocarrires for Cancer Therapy. Adv Drug Deliv Rev 66: 110116, 2014.

22 Shahbazi R, Ozpolat B and Ulubayram K: Oligonucleotide-based theranostic nanoparticles in cancer therapy, Nanomedicine (Lond) 11: 1287-1308, 2016.

23 Tekedereli I, Alpay SN, Akar U, Yuca E, Ayugo-Rodriguez C, Han HD, Sood AK, Lopez-Berestein G and Ozpolat B: Therapeutic Silencing of Bcl-2 by Systemically Administered siRNA Nanotherapeutics Inhibits Tumor Growth by Autophagy and Apoptosis and Enhances the Efficacy of Chemotherapy in Orthotopic Xenograft Models of ER (-) and ER (+) Breast Cancer. Mol Ther Nucleic Acids 2: e121, 2013.

24 Wu Y, Crawford M, Mao Y, Lee RJ, Davis IC, Elton TS, Lee LJ and Nana-Sinkam SP: Therapeutic delivery of microRNA-29b by cationic lipoplexes for lung cancer. Mol Ther Nucleic Acids 2: e84, 2013.

25 Ohno S, Takanashi M, Sudo K, Ueda S, Ishikawa A, Matsuyama N, Fujita K, Mizutani T, Ohgi T, Ochiya T, Gotoh N and Kuroda M: Systemically injected exosomes targeted to EGFR deliver antitumor microRNA to breast cancer cells. Mol Ther 21: 185$191,2012$. 
26 Jopling CL, Yi M, Lancaster AM, Lemon SM and Sarnow P: Modulation of hepatitis $\mathrm{C}$ virus RNA abundance by a liverspecific MicroRNA. Science 309: 1577-1581, 2005.

27 Pramanik D, Campbell NR, Karikari C, Chivukula R, Kent OA, Mendell JT and Maitra A: Restitution of tumor suppressor microRNAs using a systemic nanovector inhibits pancreatic cancer growth in mice. Mol Cancer Ther 10: 1470-1480, 2011.

$28 \mathrm{Hu}$ QL, Jiang QY, Jin X, Shen J, Wang K, Li YB, Xu FJ, Tang GP and Li ZH: Cationic microRNA-delivering nanovectors with bifunctional peptides for efficient treatment of PANC-1 xenograft model. Biomaterials 34: 2265-2276, 2013.

29 Arora S, Swaminathan SK, Kirtane A, Srivastava SK, Bhardwaj A, Singh S, Panyam J and Singh AP: Synthesis, characterization, and evaluation of poly (D,L-lactide-co-glycolide)-based nanoformulation of miRNA-150: potential implications for pancreatic cancer therapy. Int J Nanomedicine 9: 2933-2942, 2014.

30 Li Y and Sarkar FH: MicroRNA Targeted Therapeutic Approach for Pancreatic Cancer. Int J Biol Sci 12: 326-337, 2016.

31 Pai P, Rachagani S, Are C and Batra SK: Prospects of miRNAbased therapy for pancreatic cancer. Curr Drug Targets 14: 1101$1109,2013$.
32 Janssen HL, Reesink HW, Lawitz EJ, Zeuzem S, RodriguezTorres M, Patel K, van der Meer AJ, Patick AK, Chen A, Zhou Y, Persson R, King BD, Kauppinen S, Levin AA and Hodges MR: Treatment of HCV infection by targeting microRNA. N Engl J Med 368: 1685-1694, 2013.

33 Bader AG: miR-34-A microRNA replacement therapy is headed to the clinic. Front Genet 3: 120, 2012.

34 Seto AG, Beatty X, Lynch JM, Hermreck M, Tetzlaff M, Duvic $\mathrm{M}$ and Jackson AL: Cobomarsen, an oligonucleotide inhibitor of miR-155, co-ordinately regulates multiple survival pathways to reduce cellular proliferation and survival in cutaneous T-cell lymphoma. Br J Haematol 183: 428-444, 2018.

Received December 24, 2018

Revised January 6, 2019

Accepted January 17, 2019 